\title{
$\mathrm{ICONO} 14$
}

ICONO 14, Revista de comunicación y tecnologías emergentes, 20(1), 2022. ISSN: 1697-8293 https://doi.org/10.7195/ri14.v20i1.1752

\section{La aparición de menores en contenidos comerciales y su efecto negativo en la audiencia: el sharenting y la youtuber Verdeliss}

The presence of minors in commercial content and its negative effect on audience: sharenting and the youtuber Verdeliss

A presença de menores em conteúdos comerciais e o seu efeito negativo no público: o sharenting e youtuber Verdeliss

Angeriñe Elorriaga-Illera ${ }^{1}(\mathbb{D}$

Sergio Monge-Benito ${ }^{2}$ (D)

Elena Olabarri-Fenández ${ }^{3}$ (iD

${ }^{1}$ Profesora Adjunta (UPV/EHU), España

${ }^{2}$ Profesor Agregado (UPV/EHU), España

${ }^{3}$ Catedrática de Universidad (UPV/EHU), España

Recibido: 27/05/2021; Revisado: 14/07/2021; Aceptado: 04/11/2021; Publicado: 01/01/2022

Para citar este artículo: Elorriaga Illera, A., Monge Benito, S., Olabarri Fernández, E. (2022). La aparición de menores en contenidos comerciales y su efecto negativo en la audiencia: el sharenting y la youtuber Verdeliss, Icono 14, 20(1). https://doi.org/10.7195/ri14.v20i1.1752 


\section{Resumen}

Este artículo analiza la reacción de la audiencia de la youtuber española Verdeliss, con la mayor comunidad de seguidoras en España en ámbito de la crianza y la maternidad, en relación a la presencia de sus hijas e hijos menores en los contenidos que publica en su canal de YouTube. En una encuesta realizada a 1.336 seguidoras, se constató que más de la mitad de las mismas se sienten incómodas por la presencia de menores en videos en los que además se promocionan marcas comerciales. Este grupo valora peor a la youtuber en casi todas las dimensiones relacionadas con su capacidad persuasiva y además compra menos productos recomendados por ella en sus vídeos. Asimismo, en un análisis cualitativo de 284 comentarios en preguntas de respuesta abierta se pudo apreciar que existen varios argumentos dentro de este grupo de seguidoras más críticas con la youtuber, entre los que destacan su malestar por la excesiva exposición de los menores, un rechazo frontal a la mercantilización de la imagen de estos y un llamamiento a las instituciones competentes a que protejan a los menores. A la vista de las diferencias en la valoración de la youtuber, los resultados sugieren que el sharenting puede producir efectos negativos en la promoción de marcas comerciales.

Palabras clave: sharenting; sobreexposición; influencers; youtubers; publicidad; marcas

\section{Abstract}

This article analyzes the reaction of the audience of the Spanish youtuber Verdeliss, who has the largest community of Spanish followers in the field of parenting and motherhood, to the presence of her underage children in the contents that she publishes on YouTube. A survey made among 1.336 followers of her YouTube channel found that the majority of them feel uncomfortable because of the presence of minors in videos where commercial brands are also promoted. This group rates the youtuber worse in almost all dimensions related to her persuasive ability, and they also buy fewer products recommended by her. Likewise, in a qualitative analysis of 284 comments to open-ended questions, it was observed that there are several arguments within this group of uncomfortable followers, among which stand out their discomfort with the excessive exposure of minors, a frontal rejection to the commodification of their image, and a call on competent authorities to protect the minors. Taking into account the differences in the evaluation of the youtuber, these results suggest that sharenting could produce negative effects in the promotion of brands.

Keywords: sharenting; overexposure of minors; influencers; youtubers; advertising; brands

\section{Resumo}

Este artigo discute a reação da audiência da youtuber espanhola Verdeliss, com a maior comunidade de seguidoras em Espanha no campo da parentalidade e da maternidade, em relação à presença das suas filhas e filhos menores nos conteúdos publicados no seu canal de YouTube. Em nossa pesquisa realizada a 1.336 seguidoras, verificou-se que a majoria deles se sentem desconfortáveis com a presença de menores em vídeos que também promovem marcas comerciais. Este grupo availa a youtuber pior em quase todas as dimensões relacionadas com a sua capacidade persuasiva e também compra menos produtos recomendados por ela nos seus vídeos. Igualmente, em uma análise qualitativa de 284 comentários em questões abertas mostrou que existem vários argumentos dentro deste grupo de seguidoras mais críticas com a youtuber, entre os quais se destaca o seu mal-estar sobre a exposição excessiva de menores, uma rejeição frontal da mercantilização da sua imagem e um apelo às instituições competentes para que protegem os 
menores. Tendo em conta as diferenças na avaliação da youtuber, os resultados sugerem que o sharenting pode produzir efeitos negativos na promoção de marcas comerciais.

Palavras-chave: sharenting; sobre-exposição; influenciadores; youtubers; publicidade; marcas

\section{Introducción}

La práctica del sharenting, término acuñado para denominar la tendencia de compartir contenidos (fotos y vídeos) de hijos menores de edad en redes sociales, está muy extendida. El término se atribuye a The Wall Street Journal, como derivado de la palabra "oversharenting", que contenía una mezcla de las expresiones "overshare" y "parenting". Muchos progenitores están creando una huella digital de sus hijos, construida sin el permiso de los menores, lo que conlleva su sobreexposición en las redes sociales. Dicha sobreexposición puede atraer numerosos peligros. Un estudio de la AAP (American Academy of Pediatrics) publicado en National Conference \& Exhibition de San Francisco de 2016 afirmaba que el $50 \%$ de las imágenes de las webs pedófilas se obtienen a través de las redes sociales.

Davis (2015) realizó un estudio a una muestra estadounidense con hijos entre 0 y 4 años que concluyó que las madres y padres que utilizan las redes sociales han visto con frecuencia a otros progenitores publicar información vergonzosa sobre sus hijos (56\%), ofrecer datos personales que podrían utilizarse para identificar su localización (51\%) o compartir fotos inapropiadas de sus hijos (27\%). Según el mismo estudio, el 56\% de las madres y el $34 \%$ de los padres compartía contenidos relativos a la crianza. Otros trabajos confirman que el sharenting es una práctica más extendida entre las madres que entre los padres (Ammari et al., 2015; Duggan et al., 2015; Lazard et al., 2019).

En Turquía, Marasli et al. (2016) utilizaron una combinación de análisis de contenido con cuestionarios a los progenitores para concluir que el fenómeno del sharenting está muy presente. Un estudio británico (Nominet, 2016) descubrió que, de media, los niños británicos tienen 1500 fotografías publicadas online para cuando cumplen 5 años y en EE.UU. el 92\% de los menores de dos años ya tienen una huella digital (Jo Sales, 2016).

La legislación relativa a esta cuestión varía según el país. La ley española reconoce, por ejemplo, que "los niños son personas titulares de derechos, como el de la propia imagen" consagrado en el artículo 18.1 de la Constitución. Compartir contenidos digitales sobre los menores a tu cargo puede convertirse en un problema legal en el que entran en conflicto tu derecho a compartir lo que consideres oportuno en relación a tus hijos con el derecho de los menores de entrar en la edad adulta con la capacidad de crear su propia huella digital sin condicionamientos previos (Steinberg, 2016). Estos últimos podrían demandar a sus tutores si aprecian vulneración de sus derechos, como por ejemplo la publicación de imágenes dañinas para la propia imagen. 
Este conflicto doméstico toma una dimensión muy diferente cuando quien comparte contenidos es conocido y tiene una comunidad de seguidores de gran tamaño, ya sea una celebrity (persona previamente conocida por su actividad en la moda, el deporte, la televisión...) o una influencer de nueva generación que ha pasado de ser desconocida a volverse popular a través de sus contenidos en las redes sociales. Y el dilema ético se complejiza aún más cuando los influencers son pagados por marcas comerciales para aparecer en el mismo contenido en el que aparecen menores. La figura del menor actor de la televisión lleva mucho tiempo implantada y tiene una protección específica en la legislación, pero en las redes sociales no ocurre lo mismo.

Este artículo indaga en la reacción de la audiencia de la youtuber Verdeliss, referente en el ámbito de la crianza y la maternidad, con respecto a la exposición de los menores en los contenidos con presencia de marcas comerciales. Verdeliss (http://bit.ly/3aNMxVP) es el nombre por el que se conoce a Estefanía Unzu Ripoll, madre española de 7 menores y youtuber con más de 2 millones de seguidores. En su canal, comparte contenidos relativos a la maternidad y la crianza, incluyendo consejos relativos al embarazo, y momentos privados de la vida de su familia. Su video más visto tiene más de 20 millones de visualizaciones y tanto sus 7 hijos menores como su marido aparecen prácticamente en casi todos sus videos. Como influencer profesional, la aparición de publicidad pagada es muy frecuente. El caso es especialmente relevante porque Verdeliss es la influencer de maternidad y crianza con mayor número de seguidores en España.

El objetivo general de esta investigación es averiguar si la audiencia de Verdeliss reacciona negativamente a la presencia de menores en contenidos donde se promocionan marcas comerciales. Dicho objetivo general se materializa en los siguientes objetivos concretos:

1)Conocer las diferencias que puedan existir entre los seguidores de Verdeliss que declaran haberse sentido incomodadas por la presencia de niños en contenidos comerciales y los que no en relación a la capacidad persuasiva de la misma.

2)Identificar qué cuestiones concretas incomodan a la audiencia de la popular youtuber en relación a la aparición de menores.

\subsection{Sharenting}

El sharenting es un campo de estudio en el que convergen varios puntos de vista. Existe toda una tradición europea en torno a los riesgos y oportunidades de los menores en Internet, que ha recibido impulso de redes europeas como el Proyecto EU Kids Online (Livingstone, 2009; Livingstone, 2011), y que también ha tenido su propio desarrollo en España (Garmendia-Larrañaga et al., 2016; Jiménez et al., 2018; Garmendia et al., 2019). No obstante, son investigaciones que se han enfocado más en riesgos y oportunidades 
externos a la propia familia y no tanto a los peligros que los propios padres podrían provocar.

Otros autores que han analizado más específicamente el sharenting han detectado voces críticas o reflexivas entre los propios padres. Blum-Ross y Livingstone (2017) detectaron preocupación entre los progenitores en torno a la huella digital que genera alrededor de los menores. Otro estudio (Brosch, 2016) confirma la preocupación por la huella digital de los menores y añade otras, como identificar los límites de los derechos de los progenitores o el problema de los contenidos vergonzantes (fotos que incluyen, en algunos casos, desnudos de menores).

\subsection{Influencers y la colaboración con marcas comerciales}

Por otro lado, en el caso de Verdeliss, se añaden al debate propio de los padres que practican el sharenting su trabajo como influencer y los acuerdos comerciales que realiza con las marcas para promocionar productos. Un estudio (Elorriaga y Monge, 2018) calculó que casi la mitad $(47,6 \%)$ de los videos que publicaba la youtuber contenían referencias a marcas comerciales y el $83 \%$ de estas corresponden a apariciones pagadas.

La aparición de contenidos pagados en los influencers es una tendencia en alza en España y así lo confirman varios estudios académicos (Sanz-Marcos et al., 2019; Castelló-Martínez et al., 2015) e, incluso, estudios realizados desde el ámbito empresarial (Bandmaniac, 2018; Socialpubli.com, 2019). Estudios que han comparado la capacidad persuasiva de celebrities convencionales con influencers que se han hecho famosas a través de las redes sociales (Schouten et al., 2020; Jin et al., 2019) parecen señalar que la balanza se decanta a favor de las segundas puesto que inspiran mayor confianza y son percibidas como más similares.

A la hora de decidir con qué influencer realizar una promoción, el tamaño de la comunidad de seguidores parece el factor clave a la hora de cuantificar el efecto sobre las ventas (Zhang et al., 2018). Pero otros factores también se han estudiado en relación al efecto que tienen sobre la intención de compra de la audiencia o la evaluación del influencer: la identificación de la publicidad, y las características atribuidas a la fuente (confiabilidad, atractivo, simpatía, conocimiento experto). En relación a la primera, varios estudios han examinado el impacto negativo de identificar el contenido publicitario en la intención de compra (Evans et al., 2017; van Reijmersdal et al., 2016), en la intención de compartirlo (Lee et al., 2016; Wojdynski y Evans, 2016). No obstante, otros estudios presentan indicios contrapuestos. En el estudio de Lee et al. (2016) mencionado, el impacto negativo de identificar la publicidad no afectaba por igual a todos: los más afectados eran aquellos con mayor conocimiento persuasivo o mayor escepticismo hacia la publicidad. En un estudio cualitativo, Jiang et al. (2017) descubrieron que algunos participantes tenían percepciones positivas de la publicidad nativa porque la entendían útil, relevante e interesante. Carr y Hayes (2014) concluyeron que la percepción de credibilidad del 
influencer .blogger en su caso) era mayor cuando confesaba abiertamente el interés de terceras partes en el mensaje.

Por otro lado, en relación a la segunda, se ha apuntado en una serie de estudios previos que examinan las características de la fuente con celebrities tradicionales (Kamins, 1990, Kamins et al. 1994; Erdogan, 1999; Erdogan et al., 2001; Amos et al. 2008) que ciertos factores tienen influencia sobre la capacidad persuasiva: la honestidad/confiabilidad (trustworthiness), el conocimiento experto en un ámbito concreto (expertise), el atractivo (físico, por similaridad o simplemente simpatía o liking), o el encaje (fit, match up) entre recomendador y producto recomendado. Gran parte de la literatura disponible se refiere a celebrities convencionales, aunque existen estudios más recientes con influencers digitales que vienen a corroborar la importancia de las mismas características (Vijay et al., 2017; Martensen et al., 2018; Hughes et al., 2019).

Por último, existen algunos estudios relativos a la producción de contenidos y aparición de marcas en canales de YouTube en los que el protagonista principal es un menor (TurViñes et al., 2018; Vozmediano et al., 2018; Lopez-Villafranca, 2019; Tur-Viñes et al., 2019). Son los llamados youtubers menores o youtubers adolescentes como, por ejemplo: Las ratitas (https://bit.ly/2xiXdt3), La diversión de Martina (http://bit.ly/2RYccSP) o Mikeltube (h ttp://bit.ly/3186WjY). Los menores que aparecen en estos canales también promocionan productos, pero la mayoría de la audiencia de estos canales es menor de edad, por lo que es improbable que el debate que genera el sharenting exista también entre estos seguidores o tenga cualquier efecto sobre la capacidad persuasiva de dichos youtubers. Sin embargo, en aquellos canales dedicados a la maternidad y la crianza, dónde la audiencia es adulta, tiene potencial para generar un efecto diferente que en estos otros canales.

Por lo tanto, la literatura académica no ha explorado suficientemente el efecto que pueda tener la aparición de menores en videos promocionales sobre la capacidad persuasiva de los influencers. La maternidad y la crianza, tema central de los videos de Verdeliss, no ha sido estudiado muy en profundidad, puesto que los influencers de más éxito trabajan temas como los videojuegos, la tecnología, la moda, la belleza o el humor. Por lo tanto, no existen estudios específicos que investiguen cuál es el efecto que pueda tener los debates que surgen en torno al sharenting en la reacción de la audiencia. Los hijos e hijas de Verdeliss aparecen en la práctica totalidad de sus videos, donde muy frecuentemente se promocionan marcas comerciales cobrando por ello.

En consecuencia, cabe preguntarse, en este caso... ¿existe una reacción negativa por parte de la audiencia ante la aparición de menores en videos en los que se promocionan marcas comerciales? Para resolver esta cuestión, se plantean la siguiente hipótesis:

Hipótesis 1: La mayoría de la audiencia de esta influencer se siente incómoda con la aparición de menores en los vídeos en los que se promocionan productos. 
Por otro lado, aunque los seguidores de Verdeliss se sientan molestos, eso no necesariamente tiene por qué tener una repercusión negativa sobre su persona o las marcas que promociona. ¿Cuál es el efecto que tiene el rechazo (de existir) de la audiencia a la aparición de menores en contenidos comerciales?

Hipótesis 2: Aquellas que señalan negativamente la exposición de los menores valoran peor la capacidad persuasiva de la influencer y se ven menos influidas a comprar productos promocionados.

Finalmente, ¿cuáles son las fuentes de preocupación de la audiencia que rechaza esta práctica? Una cuestión que se ha debatido en la literatura estudiada es la huella digital de los menores y cómo en un futuro cercano dichos menores podrían sentirse molestos con la imagen que se ha generado sobre ellos.

Hipótesis 3: La huella digital de los menores es el principal argumento crítico en la audiencia de esta influencer.

\section{Material y Métodos}

Los datos fueron recogidos mediante un cuestionario auto-administrado que permaneció abierto entre el 20 de febrero de 2018 y 31 de marzo de 2018. Las participantes del estudio fueron contactadas a través del foro denominado cotilleando.com, que tiene un hilo específico sobre Verdeliss que en aquel momento tenía más de 1000 páginas (hoy en día son más de 6000). Los administradores del foro accedieron a distribuir la llamada a la participación y el único incentivo que se ofreció a los participantes fue compartir los resultados del cuestionario en ese mismo foro.

Se recibieron 1.336 respuestas válidas a lo largo de esos 40 días (se eliminaron respuestas sospechosas de haber sido rellenadas al azar o respuestas de personas que decían que nunca habían visto un video de la youtuber). Aunque normalmente este tipo de encuestas no permanecen abiertas en un periodo tan prolongado, se decidió mantenerla abierta por ese periodo extendido para recoger el máximo de respuestas en las preguntas abiertas y poder contar con mayor número de puntos de vista para el análisis cualitativo.

Durante la exploración preliminar de las respuestas abiertas de la encuesta, se detectó una fuerte presencia de comentarios contrarios a la aparición de menores en contenidos con productos patrocinados. Este artículo realiza un análisis cualitativo de dichos comentarios y lo complementa con un análisis cuantitativo de la percepción diferencial que tienen dos grupos dentro de esta audiencia: aquellas que se declaran incomodadas con la presencia de menores en esos contenidos y aquellas que no lo hacen. 
En relación a los materiales y métodos empleados, las respuestas se recogieron mediante un cuestionario de Google Forms. Los datos fueron procesados mediante el programa SPSS de IBM. Para comprobar si existía diferencia significativa entre los dos grupos (encuestadas molestas y no molestas), se emplearon pruebas t de Student para muestras independientes para averiguar si las diferencias en la medias entre dos casos eran significativas, y pruebas de Chi-cuadrado de Pearson, una prueba no paramétrica que se utiliza para probar la independencia de dos variables entre sí mediante tablas de contingencia (los valores $\mathrm{p}$ se ofrecen en las tablas correspondientes y una diferencia se considera significativa cuando el p-valor es inferior a 0,05).

En relación a las características demográficas de la muestra, las encuestadas tienen una media de $30 \pm 7,7$ años (mediana 29, mínimo 16, máximo 60), son mujeres (99,6\%), y españolas (95,6\%). El 37,5\% son madres, un 6,3\% están en proceso de serlo, y la mayoría no tiene hijos (56,2\%). Esta distribución deja claro que los contenidos sobre maternidad no interesan solo a madres, sino también a aquellas que podrían serlo en el futuro o incluso simplemente a aquellas con curiosidad sobre la vida de la youtuber. El 57,6\% tiene un empleo en el momento de realizar la encuesta $(42,4 \%$ no lo tiene, lo que sugiere por la edad que sean estudiantes). El 36,5\% visiona casi todos los videos, el 10,6\% al menos la mitad, el $6,4 \%$ al menos un cuarto, y el $20,5 \%$ solo algún video suelto. También hay un porcentaje considerable $(25,9 \%)$ que ha dejado de ver sus videos, aunque los vio en algún momento. Se desestimaron las respuestas de aquellas que declararon no haber visto nunca un video suyo. Cuando se les pregunta por su posición hacia la youtuber, el 21,6\% se declara detractora de Verdeliss, un 5,7\% defensora de ella, mientras que un $47,1 \%$ se considera neutral y un $25,6 \%$ no se lo ha planteado.

\section{Resultados}

Para comprobar las hipótesis de este artículo, se dividió la muestra entre aquellas seguidoras que declararon sentirse cómodas con los contenidos en los que aparecían menores y aquellas que declararon su incomodidad al respecto. Para realizar dicha división, se utilizaron dos preguntas de la encuesta. La primera de ellas cuestionaba si se habían sentido incómodas alguna vez viendo videos en los que aparecían marcas comerciales ("¿Te has sentido en alguna ocasión incómoda viendo los vídeos de Verdeliss en los que aparecen marcas comerciales?"). El 77,5\% respondió afirmativamente. Después de responder esta primera pregunta, se preguntaban los motivos de tal incomodidad, y el $67,3 \%$ de aquellas que se habían sentido incómodas estaba de acuerdo (4) o completamente de acuerdo (5) con que no deberían aparecer menores en los videos en los que aparecían marcas comerciales. En total, si se toma la muestra al completo, un 52,2\% se declara incómoda porque aparezcan conjuntamente menores y marcas comerciales, mientras que el otro $47,8 \%$ no muestra esta misma incomodidad por esa cuestión concreta. Esos son los dos grupos de encuestadas, que hemos denominado posteriormente como "molestas" y "no molestas", respectivamente. 
Esto permite confirmar la primera hipótesis, "La mayoría de la audiencia de esta influencer se siente molesta con la aparición de menores en los vídeos en los que se promocionan productos", si bien por un estrecho margen de 52,2\% contra 47,8\%. La audiencia de Verdeliss se encuentra polarizada en relación a este tema y, como veremos a continuación, esa polarización hace que perciban a la youtuber de manera diferente.

La Tabla 1 muestra las diferencias en las características entre estos dos grupos ( $p$ valor menor que 0,05 es significativo en la prueba correspondiente, sea t de Student o Chi-cuadrado). Aunque aparecen diferencias significativas por el tamaño de la muestra, las diferencias de edad son muy pequeñas. Aquellas que se declaran incómodas con la presencia de los menores son apenas un año mayores que las que no $(30,58$ años frente a 29,46). No existen diferencias significativas en la proporción de madres (se observa también en la Tabla 1) ni tampoco en otras variables que no están incluidas en la tabla, como el empleo o el sexo (ya hemos declarado que el $99,6 \%$ son mujeres). Por supuesto, todas las que están molestas por la presencia de menores y marcas se han declarado "incómodas" con la publicidad, ya que era precisamente el factor de selección. Sin embargo, en el grupo de las que no se declaran incómodas por la cuestión de los menores, hay un $53 \%$ que muestra incomodidad con la publicidad presente en los contenidos por otros motivos no relacionados con los menores, como pueden ser la excesiva presencia de publicidad, la aparición forzada de productos, o la poca relación de los productos con la experiencia de la youtuber. 
Tabla 1. Diferencias entre molestas y no molestas con la presencia de videos en los que aparecen menores y marcas

\begin{tabular}{|l|c|c|c|}
\hline & Molestas & No molestas & $\begin{array}{c}\text { iSignificativo? } \\
\text { (p-valor) }\end{array}$ \\
\hline Muestra & $698(52,2 \%)$ & $638(47,8 \%)$ & n/a \\
\hline Edad & $30,58 \pm 7,9$ & $29,46 \pm 7,6$ & Sí $(0,008)$ \\
\hline $\begin{array}{l}\text { ¿Es madre? Sí/En } \\
\text { proceso/No }\end{array}$ & $38,4 \% / 6,3 \% / 55,3 \%$ & $36,5 \% / 6,3 \% / 57,2 \%$ & No $(0,85)$ \\
\hline $\begin{array}{l}\text { ¿Se ha sentido } \\
\text { incómoda alguna vez } \\
\text { con los videos que } \\
\text { incorporan marcas } \\
\text { comerciales? }\end{array}$ & $100 \%$ & $53 \%$ & n/a \\
\hline
\end{tabular}

Fuente: elaboración propia a partir de los resultados de la encuesta

No obstante, cuando se revisan en la Tabla 2 las valoraciones que otorgan a Verdeliss en varias dimensiones relacionadas con su capacidad persuasiva (similaridad, atractivo físico, liking, respeto, honestidad y su credibilidad recomendando la práctica totalidad de los productos), se observa que aquellas seguidoras molestas con el uso que hace de sus hijos valoran significativamente peor a la youtuber en todas las categorías (utilizando una prueba t para muestras independientes). Se trata de un efecto robusto, que se mantiene en todas las dimensiones de la evaluación excepto en la familiaridad con la youtuber. En ese caso particular, también existe diferencia significativa, pero el grupo que se declara incómodo con la aparición de menores responde tener mayor grado de conocimiento sobre la vida de la influencer (mayor familiaridad, 3,41 vs 3,09) que el grupo no incómodo por esta cuestión. 
Tabla 2. Diferencias en la evaluación de características de la fuente Verdeliss (en una escala 1-5) entre aquellas molestas (se declaran "incómodas") y aquellas no molestas con la aparición de menores en los videos que promocionan marcas

\begin{tabular}{|l|l|l|l|}
\hline Característica evaluada & Molestas & $\begin{array}{l}\text { No } \\
\text { molestas }\end{array}$ & $\begin{array}{l}\text { ¿Significativo? } \\
\text { (p-valor) }\end{array}$ \\
\hline $\begin{array}{l}\text { Considero que Verdeliss es similar a mí } \\
\text { en muchos aspectos (similaridad) }\end{array}$ & $1,74 \pm 0,81$ & $2,24 \pm 1,01$ & Sí $(0,000)$ \\
\hline $\begin{array}{l}\text { Considero que Verdeliss es una mujer } \\
\text { atractiva físicamente (atractivo físico) }\end{array}$ & $2,60 \pm 1,06$ & $3,06 \pm 1,07$ & Sí $(0,000)$ \\
\hline $\begin{array}{l}\text { Considero que tengo un alto grado de } \\
\text { conocimiento sobre la vida de Verdeliss } \\
\text { (familiaridad) }\end{array}$ & $3,41 \pm 1,18$ & $3,09 \pm 1,15$ & Sí $(0,000)$ \\
\hline $\begin{array}{l}\text { Considero que Verdeliss es una de mis } \\
\text { favoritas (liking) }\end{array}$ & $1,89 \pm 0,94$ & $2,67 \pm 1,23$ & Sí $(0,000)$ \\
\hline $\begin{array}{l}\text { Considero que Verdeliss merece todo } \\
\text { mi respeto (respeto) }\end{array}$ & $2,67 \pm 1,18$ & $3,44 \pm 1,24$ & Sí $(0,000)$ \\
\hline $\begin{array}{l}\text { Verdeliss no tiene motivos para mentir } \\
\text { a su audiencia (honestidad) }\end{array}$ & $2,35 \pm 1,24$ & $2,92 \pm 1,35$ & Sí $(0,000)$ \\
\hline $\begin{array}{l}\text { Para mí, Verdeliss conoce y resulta } \\
\text { creíble recomendando productos de: } \\
\text { (Credibilidad) }\end{array}$ & & & \\
\hline
\end{tabular}




\begin{tabular}{|l|l|l|l|}
\hline -Alimentación & $1,70 \pm 0,91$ & $2,68 \pm 1,20$ & Sí $(0,000)$ \\
\hline -Limpieza & $1,90 \pm 0,97$ & $2,79 \pm 1,16$ & Sí $(0,000)$ \\
\hline -Libros & $1,94 \pm 0,99$ & $2,85 \pm 1,27$ & Sí $(0,000)$ \\
\hline -Juguetes & $2,31 \pm 1,13$ & $3,18 \pm 1,19$ & Sí $(0,000)$ \\
\hline -Cosmética & $1,40 \pm 0,72$ & $2,16 \pm 1,09$ & Sí $(0,000)$ \\
\hline -Destinos Vacacionales & $1,67 \pm 0,89$ & $2,49 \pm 1,17$ & Sí $(0,000)$ \\
\hline -Farmacia & $1,77 \pm 0,93$ & $2,59 \pm 1,18$ & Sí $(0,000)$ \\
\hline -ONGs & $1,64 \pm 0,93$ & $2,66 \pm 1,29$ & Sí $(0,000)$ \\
\hline -Moda & $1,46 \pm 0,73$ & $2,28 \pm 1,12$ & Sí $(0,000)$ \\
\hline -Decoración & $1,56 \pm 0,82$ & $2,47 \pm 1,15$ & Sí $(0,000)$ \\
\hline -Aplicaciones/webs & $1,50 \pm 0,77$ & $2,32 \pm 1,14$ & Sí $(0,000)$ \\
\hline -Productos Infantiles & $3,20 \pm 1,27$ & $3,89 \pm 1,02$ & Sí $(0,000)$ \\
\hline
\end{tabular}

Fuente: elaboración propia a partir de los resultados de la encuesta

Además de la peor valoración de la figura de la youtuber que nos muestra la Tabla 2, existe diferencia significativa (Chi-cuadrado de Pearson) también en el porcentaje de ellas que declara haber comprado algún producto recomendado: un 7\% entre aquellas seguidoras molestas por la aparición de menores con marcas comerciales frente a un $16 \%$ de las no molestas (Tabla 3). 
Tabla 3. Porcentaje de las encuestadas que declara haber comprado productos en función de si se declaran incómodas ("molestas") con la aparición de menores en publicaciones con presencia de marcas comerciales

\begin{tabular}{|l|l|l|l|}
\hline & Molestas & No molestas & $\begin{array}{l}\text { ¿Significativo? } \\
\text { (p-valor) }\end{array}$ \\
\hline $\begin{array}{l}\text { Ha comprado productos } \\
\text { recomendados por Verdeliss }\end{array}$ & $7 \%$ & $16 \%$ & Sí $(0,000)$ \\
\hline
\end{tabular}

Fuente: elaboración propia a partir de los resultados de la encuesta

Con las evaluaciones de Verdeliss (Tabla 2) y los porcentajes de compra de productos (Tabla 3), se puede confirmar que la segunda hipótesis también se cumple: "aquellas que señalan negativamente la exposición de los menores valoran peor la capacidad persuasiva de la influencer y se ven menos influidas a comprar productos promocionados". La única excepción al cumplimiento de esta hipótesis se produce en la dimensión familiaridad: las seguidoras molestas declaran conocer más a fondo la vida de la youtuber que aquellas no molestas.

Por último, para resolver la tercera hipótesis es necesario revisar las respuestas a las dos preguntas abiertas de la encuesta. La primera la podían responder aquellas seguidoras que se habían sentido molestas con la aparición de marcas comerciales y querían aclarar algo más al respecto. La segunda era una pregunta abierta al final de la encuesta, en la que se preguntaba si había algo que no se le hubiera preguntado sobre lo que quisiera comentar algo.

En total, se recibieron 284 comentarios diferentes (teniendo en cuenta ambas preguntas abiertas). De entre ellos, 147 (51,7\% del total) expresaron malestar con la publicidad clandestina de la youtuber, con la falta de credibilidad y con la presencia excesiva de publicidad, cada una de ellas con un número similar de opiniones. Los comentarios positivos son una excepción: 15 testimonios (5,3\% del total) alaban el trabajo de la youtuber sin atisbo de crítica.

Para confirmar o desmentir la tercera hipótesis debemos revisar el resto de comentarios, 137 (48,2\% del total), que hacen referencia a los menores en los videos de Verdeliss, expresando diferentes tipos de quejas que podemos clasificar en cuatro líneas argumentales (algunos de los comentarios se clasifican en más de una línea argumental): 
La primera de estas líneas argumentales (70 comentarios, el $51 \%$ de los 137) la componen los comentarios que aluden a la mercantilización de la que son objeto los menores. Los comentarios describen una nueva forma de explotación infantil encubierta, disfrazada de cotidianidad, que consiste en lucrarse de la exhibición de la privacidad de los menores: "Me parece lamentable tener hijos para usarlos como mercancía y vender sus vidas para ganar dinero y que los padres no tengan que trabajar. Basta de esta nueva explotación infantil encubierta". La audiencia considera que sus hijos son utilizados para crear contenidos: "Hace que sus hijos formen parte de un guion para vender productos o ponerlos en situaciones que den pie a publicitar uno", incluso obligados: "Me parece muy triste ver a unos niños forzar una sonrisa solo porque su madre lo exige para ganar dinero". Les expone incluso cuando el producto anunciado nada tiene que ver con ellos: "El problema es la utilización de menores para conseguir dinero de las marcas, por ejemplo, publica en instagram una foto de dos niñas menores de 2 años con un jamón". Los comentarios describen la práctica habitual de la youtuber de servirse de sus descendientes como reclamo para atraer visitas y la acusan de convertir la vida de estos en un negocio: "Sigo viéndolos porque los críos son un encanto, pero están completamente vendidos".

Relacionada con la anterior y con un número de opiniones muy próximo (66 comentarios, el 48,2\% de los 137), la segunda línea argumental hace referencia a la sobreexposición de los menores. Se alude a la exhibición irresponsable de los mismos sin ningún tipo de control o de razón: "Sobre todo me preocupa la exposición de sus hijos tan aleatoria, tan constante, tan porque sí... Creo que es lo que más preocupa a las lectoras de este foro". A su juicio, se trata de una exposición constante, excesiva, de las vidas de sus hijos: "Con lo que más en desacuerdo estoy es con que exponga la vida de sus hijos de esa manera." No aparece reflexión alguna sobre las consecuencias futuras de esta actividad: "Creo que el tema de los niños se le fue un poco de las manos, no tiene en cuenta que quizá ellos a la larga no se sientan cómodos con toda su vida íntima expuesta (...)".

La tercera línea argumental hace referencia a la exposición de los menores en posiciones de vulnerabilidad (con 28 opiniones, el 20,4\% de los 137). En sus comentarios, la pregunta que emerge tiene que ver con dónde queda la privacidad, la intimidad y el recelo de las personas que se aman. Esta parte de la audiencia describe a la influencer como una madre que no sabe o no quiere proteger a sus hijos e hijas: "Debería de controlar más cuanto enseña de sus hijos". De alguna manera, manifiestan que Verdeliss vende sus vidas, les arrebata algo que les pertenece: "Utiliza la intimidad de sus hijos menores para lucrarse" y "Está ganando dinero a base de dejar a sus niños sin privacidad alguna". Esta exposición de la intimidad, en algunos casos, presenta contenidos, a juicio de las entrevistadas, vergonzantes y escenas o situaciones en las que los menores aparecen comprometidos: "Sobreexpone a los niños especialmente en el baño, sin ropa, sabiendo que es peligroso..." o "Enseña a sus hijos comiendo, durmiendo, bañándose, jugando desnudos, las caídas, los vómitos". La audiencia es sensible a la vulnerabilidad de los mismos: "... que cualquier mal parido puede usarlo como pornografía infantil, nunca sabes quien está al otro lado de la pantalla". En esta línea, una preocupación que emerge, aunque no es mayoritaria, es la relativa a la huella digital y las consecuencias futuras de la misma en los menores: "...no 
respeta su intimidad, hecho que puede causarles algún mal en el futuro, tanto en sus relaciones personales como laborales" o "Exhibe a sus hijos sin importarle como salgan y no tiene en cuenta que a ellos el día de mañana no les guste que toda su vida esté en internet".

La cuarta línea argumental explicita que los menores tienen derechos que sus progenitores a veces olvidan, y una parte no despreciable de los que han dejado comentarios molestos reclama que esos derechos se hagan valer. Lo que se observa en esta línea argumental es un llamamiento a las instituciones para que protejan a los menores (21 opiniones, el 15,3\% de los 137). Las encuestadas buscan autoridades competentes que puedan intervenir en la situación: "(...) si los propios padres no saben o no quieren protegerlos por el hecho de utilizarlos para fines económicos (...) la ley es la que debe protegerlos". Demandan que la legislación se adecue a los nuevos tiempos e interpretan la situación de los hijos de Verdeliss como similar a la que tienen los niños actores (que sí que cuentan con legislación específica): "Protección al menor en youtube. Son niños actores que sus padres se están aprovechando de ellos económicamente (...)."

Por lo tanto, a la vista de la revisión cualitativa de estos comentarios, se observa que la tercera hipótesis, "La huella digital de los menores es el principal argumento crítico en la audiencia de esta influencer", se cumple solo parcialmente. Aunque la preocupación por la huella digital, es decir, por la cantidad de contenidos que quedan disponibles en la red a lo largo del tiempo con la imagen de esos menores, existe, esta aparece marginalmente por detrás de otras preocupaciones con mayor presencia: la mercantilización de los menores (obtención de rentas a partir de su imagen), la sobreexposición de estos, la vulneración de su intimidad, y el llamamiento a la intervención por parte de las autoridades. La huella digital es una preocupación que aparece con fuerza en la literatura científica, pero aparece eclipsada por otras cuestiones en el discurso de su audiencia.

\section{Conclusiones y discusión}

Los resultados muestran que la audiencia de Verdeliss está dividida entre quienes se declaran incómodas con la aparición de marcas comerciales en contenidos en los que aparecen menores y quienes no tienen problemas con ello (grupos que se han denominado "molestas" y "no molestas" a lo largo del texto). El primer grupo valora a la youtuber significativamente peor en casi todas las dimensiones relacionadas con su capacidad persuasiva (similaridad, honestidad, atractivo, competencia, etc.). Además, un porcentaje mucho menor (inferior a la mitad que en el grupo no molesto) declaró haber comprado productos recomendados por Verdeliss. Los argumentos principales que justifican esta percepción menos amable de la youtuber están relacionados con considerar esta forma de sharenting como una mercantilización de los hijos menores, con entender que no se protege suficientemente la intimidad de los mismos y con reclamar acción por parte de las instituciones competentes. 
Los resultados de este estudio demuestran que la aparición de menores en contenidos comerciales puede concitar rechazo de una parte de la audiencia. Este hecho debería ser tenido en cuenta por las marcas que colaboran con influencers, puesto que pueden ver mermado el efecto de sus campañas por ese efecto rechazo. Rechazo que por otra parte conecta con una tendencia social de creciente preocupación con la exposición de menores en redes sociales por parte de sus progenitores (sharenting). Un ejemplo claro de esta tendencia en negativo es la petición en la plataforma change.org que contiene una foto de Verdeliss, su marido y sus descendientes: "No a la explotación de menores en YouTube" (ht tps://www.change.org/p/protegeles-no-a-la-explotaci\%C3\%B3n-de-menores-en-youtube).

Pero la intersección entre el sharenting y la promoción de marcas es un fenómeno que no solo afecta a influencers con descendientes sino también a progenitores con hijos influencers, y así se ha observado en la revisión de la literatura que describe varios casos de menores como generadores de contenido digital con promociones de marcas (Tur-Viñes et al., 2018; Vozmediano et al., 2018; López-Villafranca y Olmedo-Salar, 2019; Tur-Viñes et al., 2019). Puesto que en estos casos las audiencias las forman mayoritariamente menores, es posible que el efecto negativo que vemos en la audiencia de Verdeliss no se reproduzca en la de estos otros youtuber. Pero las empresas que se promocionan en estos canales deberían como mínimo considerar que es una posibilidad. Además, la audiencia de estos influencers menores podría ser también objeto de una investigación futura que describiera las diferencias con los resultados presentados en este artículo.

En cualquier caso, las instituciones deberían concienciar a la sociedad de los peligros y problemas éticos que supone el sharenting. También deberían proponer buenas prácticas que traten dichos problemas de una manera adecuada. Otros ámbitos con responsabilidad que podrían declararse al respecto son las asociaciones profesionales de la comunicación y los sistemas de autorregulación del sistema publicitario como Autocontrol. Estas asociaciones podrían establecer guías de buenas prácticas al respecto. En la legislación española, por ejemplo, existen limitaciones muy claras sobre la participación de menores en el rodaje de anuncios publicitarios y películas de cine. No obstante, cuando el productor de contenido es también progenitor del menor, parece que las barreras se difuminan y desaparecen los límites legales.

A la vista de las diferencias en la valoración de la youtuber y en el porcentaje de productos comprados, detectar otras fuentes similares de rechazo en las audiencias puede resultar sumamente interesante para las marcas que practican el marketing de influencers. Identificar reacciones críticas similares en las audiencias de los influencers con los que trabajan permitiría realizar inversiones publicitarias más adecuadas y efectivas.

Por último, los resultados descritos en este artículo tienen la limitación de corresponder a un solo caso. Sería interesante ampliar los estudios de caso a otros influencers, de ámbitos distintos a la maternidad y crianza, para descubrir si existen algunos otros factores ocultos que puedan estar polarizando su audiencia. Por otro lado, padres y madres anónimos podrían estar generando reacciones negativas similares a los observados en la audiencia 
de Verdeliss al compartir contenidos de sus hijos e hijas, aunque no exista mercantilización alguna. Las reacciones de estos padres y madres al sharenting de conocidos o amigos serían, por lo tanto, otro objeto de estudio interesante con el que comparar.

\section{Referencias}

American Academy of Pediatrics (AAP) (2016). What Parents Should Share: Child Privacy in the Age of Social Media and the Pediatrician's Role. National Conference \& Exhibition, San Francisco.

Ammari, T., Kumar, P., Lampe, C. y Schoenebeck, S. (2015). Managing children's online identities: How parents decide what to disclose about their children online. Proceedings of the 33rd annual ACM conference on human factors in computing systems (pp. 1895-1904). https://doi.org/10.114 $5 / 2702123.2702325$

Amos, C., Holmes, G., y Strutton, D. (2008). Exploring the relationship between celebrity endorser effects and advertising effectiveness: A quantitative synthesis of effect size. International Journal of Advertising, 27(2), 209-234. https://doi.org/10.1080/02650487.2008.11073052

Blum-Ross, A., y Livingstone, S. (2017). "Sharenting," parent blogging, and the boundaries of the digital self. Popular Communication, 15(2), 110-125. https://doi.org/10.1080/15405702.2016.12 23300

Brandmaniac (2018). Estudio sobre Marketing de Influencers en España, 2018. Documento online (consultado 8/10/2019). http://bit.ly/38a3grt

Brosch, A. (2016). When the Child is born into the internet: sharenting as a Growing trend among Parents on Facebook. The New Educational Review, 43(1), 225-235. https://doi.org/10.15804/tn er.2016.43.1.19

Carr, C. T., y Hayes, R. A. (2014). The effect of disclosure of third-party influence on an opinion leader's credibility and electronic word of mouth in two-step flow. Journal of Interactive Advertising, 14(1), 38-50. https://doi.org/10.1080/15252019.2014.909296

Castelló-Martínez, A., y Pino, C. D. (2015). La comunicación publicitaria con influencers. Redmarka: revista académica de marketing aplicado, 14, 21-50.

Clark, L. S. (2013). The parent app: Understanding families in the digital age. Oxford, UK: Oxford University Press. https://doi.org/10.17979/redma.2015.01.014.4880

Davis, M.M. (2015). Parents on Social Media: Likes and Dislikes of Sharenting. Documento online (descargado 5/10/2019) http://bit.ly/2UI3HiO

Duggan, M., Lenhart, A., Lampe, C., y Ellison, N. (2015). Parents and social media. Pew Research Centre (Washington, DC). https://pewrsr.ch/39u5BqB

Elorriaga-lllera, A., y Monge-Benito, S. (2018). La profesionalización de los YouTubers: el caso de Verdeliss y las marcas. Revista Latina de Comunicación Social, 73, 37-54. https://doi.org/10.418 5/RLCS-2018-1244

Erdogan, B.Z. (1999). Celebrity endorsement: A literature review. Journal of Marketing Management, 15(4), 291-314. https://doi.org/10.1362/026725799784870379

Erdogan, Z., Baker, R., y Tagg, S. (2001). Selecting celebrity endorsers: the practitioner perspective. Journal of Advertising Research, 41(3), 39-48. https://doi.org/10.2501/JAR-41-3-39-48

Evans, N. J., Phua, J., Lim, J., y Jun, H. (2017). Disclosing Instagram influencer advertising: The effects of disclosure language on advertising recognition, attitudes, and behavioral intent. Journal of Interactive Advertising, 17(2), 138-149. https://doi.org/10.1080/15252019.2017.1366885 
Garmendia-Larrañaga, M., Jiménez-Iglesias, E., Casado, M.A., y Mascheroni, G. (2016). Net Children Go Mobile. Riesgos y oportunidades de internet y uso de dispositivos móviles entre menores españoles (2010-2015). Red.es. https://netchildrengomobile.eu/ncgm/wp-content/uploads/201 3/07/Net-Children-Go-Mobile-Spain.pdf

Garmendia, M., Jiménez, E., Karrera, I., Larrañaga, N., Casado, M.A., y Garitaonandia, C. (2019). Actividades, mediación, oportunidades y riesgos online de los menores en la era de la convergencia mediática. Instituto Nacional de Ciberseguridad (INCIBE). León (España).

Hughes, C., Swaminathan, V., y Brooks, G. (2019). Driving brand engagement through online social influencers: An empirical investigation of sponsored blogging campaigns. Journal of Marketing, 83(5), 78-96. https://doi.org/10.1177/0022242919854374

Jiang, M., McKay, B. A., Richards, J. I., y Snyder, W. (2017). Now you see me, but you don't know: Consumer processing of native advertisements in online news sites. Journal of Interactive Advertising, 17(2), 92-108. https://doi.org/10.1080/15252019.2017.1399839

Jiménez, E., Garmendia, M., y Casado, M.A. (2018). Entre selfies y whatsapps. Oportunidades y riesgos para la infancia y la adolescencia conectada. Gedisa. Barcelona. https://doi.org/10.14198/MED COM2018.9.2.13

Jin, S. V., Muqaddam, A., y Ryu, E. (2019). Instafamous and social media influencer marketing. Marketing Intelligence \& Planning, 37(5). https://dx.doi.org/10.1108/MIP-09-2018-0375

Jo Sales, N. (27 de marzo de 2016). American Girls. New York Times. https://nyti.ms/2OEPoHa

Kamins, M.A. (1990). An investigation into the match-up hypothesis in celebrity advertising: when beauty may be only skin deep. Journal of Advertising, 19(1), 4-13. https://doi.org/10.1080/009 13367.1990.10673175

Kamins, M.A., y Gupta, K. (1994). Congruence between spokesperson and product types: a matchup hypothesis perspective. Psychology and Marketing, 11(6), 569-586. https://doi.org/10.1002/ma r.4220110605

Lazard, L., Capdevila, R., Dann, C., Locke, A., y Roper, S. (2019). Sharenting: Pride, affect and the dayto-day politics of digital mothering. Soc Personal Psychol Compass. https://doi.org/10.1111/sp c3.12443

Lee, J., Kim, S., y Ham, C.D. (2016). A double-edged sword? Predicting consumers' attitudes toward and sharing intention of native advertising on social media. American Behavioral Scientist, 60(12), 1425-1441. https://doi.org/10.1177/0002764216660137

Livingstone, S., y Haddon, L. (Eds.). (2009). Kids online: Opportunities and risks for children. Policy press.

Livingstone, S., Haddon, L., Görzig, A., y Ólafsson, K. (2011). Risks and safety on the internet: the perspective of European children: full findings and policy implications from the EU Kids Online survey of 9-16-year-olds and their parents in 25 countries [Archivo PDF]. http://eprints.Ise.ac .uk/id/eprint/33731

López-Villafranca, P., y Olmedo-Salar, S. (2019). Menores en YouTube, ¿ocio o negocio? Análisis de casos en España y EUA. El profesional de la información, 28(5). https://doi.org/10.3145/epi.20 19.sep.20

Marasli, M., Suhendan, E., Yilmazturk, N. H., y Cok, F. (2016). Parents' shares on social networking sites about their children: sharenting. The Anthropologist, 24(2), 399-406. https://doi.org/10.10 80/09720073.2016.11892031

Martensen, A., Brockenhuus-Schack, S., y Zahid, A. L. (2018). How citizen influencers persuade their followers. Fashion Marketing and Management, 22(3), 335-353. https://doi.org/10.1108/JFMM-0 9-2017-0095 
Nominet (2016). Share with Care [Archivo PDF]. http://bit.ly/2ULb3RT

Sanz-Marcos, P., Jiménez-Marín, G., y Elías-Zambrano, R. (2019). La incorporación de la figura del influencer en las campañas publicitarias: Consecuencias para las agencias de publicidad españolas. Revista adComunica, 18, 63-86. http://dx.doi.org/10.6035/2174-0992.2019.18.5

Socialpubli.com (2019). I Estudio de Anunciantes con Influencers [Archivo PDF] http://bit.ly/31DKqj6

Steinberg, S. B. (2016). Sharenting: Children's privacy in the age of social media. Emory LJ, 66, 839.

Schouten, A.P., Janssen, L., y Verspaget, M. (2020). Celebrity vs. Influencer endorsements in advertising: the role of identification, credibility, and Product-Endorser fit. International Journal of Advertising, 39, pp. 258-281. https://doi.org/10.1080/02650487.2019.1634898

Tur-Viñes, V., Núñez Gómez, P., y González-Río, M.J. (2018). Menores influyentes en YouTube. Un espacio para la responsabilidad. Revista Latina de Comunicación Social, 73, 1211-1230. https:/ /doi.org/10.4185/RLCS-2018-1303

Tur-Viñes, V., Gómez, P. N., y Pastor, E. M. (2019). YouTube, menores y cultura colaborativa. Revisión bibliográfica de la investigación académica. Revista Historia y comunicación social, 24(1), 331-351. https://doi.org/10.5209/hics.64498

Van Reijmersdal, E., Fransen, M. L., Van Noort, G., Opree, S. J., Vandeberg, L., Reusch, S., y Boerman, S. C. (2016). Effects of disclosing sponsored content in blogs: How the use of resistance strategies mediates effects on persuasion. American Behavioral Scientist, 60(12), 1458-1474. $\underline{\mathrm{h}}$ ttps://doi.org/10.1177/0002764216660141

Vijay, T. S., Prashar, S., Parsad, C., y Kumar, M. (2017). An Empirical Examination of the Influence of Information and Source Characteristics on Consumers' Adoption of Online Reviews. Pacific Asia Journal of the Association for Information Systems, 9(1). https://doi.org/10.17705/1 pais.09104

Vozmediano, M. M., Jiménez, A. G., y Sendra, J. M. (2018). Los vídeos de los adolescentes en YouTube: Características y vulnerabilidades digitales. Comunicar: Revista científica iberoamericana de comunicación y educación, 54, 61-69. https://doi.org/10.3916/C54-2018-06

Wojdynski, B. W., y Evans, N. J. (2016). Going native: Effects of disclosure position and language on the recognition and evaluation of online native advertising. Journal of Advertising, 45(2), 157-168. https://doi.org/10.1080/00913367.2015.1115380

Zhang, Y., Lin, Y. y Goh, K. H. (2018). Impact of Online Influencer Endorsement on Product Sales: Quantifying Value of Online Influencer. En PACIS 2018 proceedings, p. 201. https://core.ac.uk/ download/pdf/301375963.pdf 